\title{
Plasma tubes becoming collimated as a result of magnetohydrodynamic pumping
}

\author{
Gunsu S. Yun ${ }^{\text {a) }}$ and Paul M. Bellan \\ California Institute of Technology, Pasadena, California 91125, USA
}

(Received 5 February 2010; accepted 6 May 2010; published online 17 June 2010)

\begin{abstract}
Collimated magnetized plasma structures are commonly observed on galactic, stellar, and laboratory scales. The Caltech plasma gun produces magnetically driven plasma jets bearing a striking resemblance to astrophysical jets and solar coronal loops by imposing boundary conditions analogous to those plasmas. This paper presents experimental observations of gun-produced plasma jets that support a previously proposed magnetohydrodynamic (MHD) pumping model [P. M. Bellan, Phys. Plasmas 10, 1999 (2003)] as a universal collimation mechanism. For any initially flared, magnetized plasma tube with a finite axial current, the model predicts (i) magnetic pumping of plasma particles from a constricted region into a bulged region and (ii) tube collimation if the flow slows down at the bulged region leading to accumulation of mass and thus concentrating the azimuthal magnetic flux frozen in the mass flow (i.e., increasing the pinch force). Time- and space-resolved spectroscopic measurements of gun-produced plasmas have confirmed the highly dynamic nature of the process leading to a collimated state, namely, (i) suprathermal Alfvénic flow $(30-50 \mathrm{~km} / \mathrm{s})$, (ii) large density amplification from $\sim 10^{17}$ to $\sim 10^{22} \mathrm{~m}^{-3}$ in an Alfvénic time scale $(5-10 \mu \mathrm{s})$, and (iii) flow slowing down and mass accumulation at the flow front, the place where the tube collimation occurs according to high-speed camera imaging. These observations are consistent with the predictions of the MHD pumping model, and offer valuable insight into the formation mechanism of laboratory, solar, and astrophysical plasma structures. (C) 2010 American Institute of Physics. [doi:10.1063/1.3437075]
\end{abstract}

\section{INTRODUCTION}

Collimated structure, i.e., long tubular morphology with constant cross-sectional area, is the most conspicuous common feature of a wide variety of magnetized plasmas, ranging from astrophysical plasma jets to solar prominences and laboratory plasmas.

Astrophysical jets are a class of bipolar mass flows emanating from massive astronomical objects such as active galactic nuclei, young stellar objects, and high-mass x-ray binaries. The mass flows extend from the central objects to the interstellar media with extremely high velocity, often relativistic. An astounding feature of the astrophysical jets is the sustainment of collimated structure over a length scale $\left(10^{15}-10^{22} \mathrm{~m}\right)$ many orders of magnitude larger than the source objects. Magnetic fields observed in some astrophysical jets ${ }^{1}$ and numerical modelings ${ }^{2}$ strongly suggest that magnetohydrodynamics (MHD) plays an important role governing jet formation and collimation. The reason for the collimation process has been somewhat of a mystery.

Solar prominences are arch-shaped magnetized plasma structures protruding from the surface of the Sun. ${ }^{3}$ Typical prominences extend over many thousands of kilometers and are often stable for many days. Plasma particles are localized inside the collimated solar prominences, and so there exists a large density gradient across the boundaries. The stability of the solar prominences despite the large density gradient has

\footnotetext{
${ }^{a)}$ Present address: Pohang University of Science and Technology, Pohang, Gyungbuk 790-784, Korea. Electronic mail: gunsu@ postech.ac.kr.
}

not been understood yet. The subcategory called coronal loops are especially known for extremely collimated magnetic flux tubes. These magnetic flux loops originate from the photosphere and extend to the corona region, and have lengths of $10^{7}-10^{8} \mathrm{~m}$. Coronal loops are suspected to be the energy transport channel responsible for the anomalous coronal heating. ${ }^{3}$ However, the origin of the extreme collimation and the observed mass flow have been a mystery.

Images of the laboratory plasmas produced by the Caltech planar coaxial plasma gun, which will be described in detail in Sec. III, show remarkable similarities with the above naturally occurring plasmas. ${ }^{4}$ The laboratory plasmas are compared with a solar prominence and an astrophysical jet in Figs. 1(a) and 1(b), respectively.

Motivated by the observations of collimated plasma structures both in nature and laboratory, Bellan ${ }^{5}$ proposed a universal MHD pumping process which explains why collimated plasma-filled magnetic flux tubes are ubiquitous. The MHD pumping model is based on the concept of frozen-in magnetic flux and effective magnetic potential energy. The model predicts Alfvénic mass flow driven by the gradient of the magnetic potential and a density amplification associated with the flow gradient. In this picture, collimation is a highly dynamic process involving MHD-driven flows constrained by frozen-in flux considerations. This process leads to the formation of a collimated plasma-filled flux tube, that is, the presumed initial condition for kink and sausage instabilities. Thus, the overall sequence is MHD-driven jet flows produce a collimated plasma-filled flux tube which may then undergo a kink or sausage instability. The kinking of a jet-produced 
collimated flux tube has been discussed in detail previously by Hsu and Bellan ${ }^{6}$ and will not be addressed here.

This paper focuses on the dynamics of laboratory plasma jets before any kinking or sausaging occurs, i.e., on the dynamics associated with collimation. In particular, the paper reports detailed measurements of density and flow velocity of the jets supporting the MHD pumping model as a universal collimation mechanism. The basic concepts of the model are summarized in Sec. II. The Caltech plasma gun is introduced along with a review of previous experimental work in Sec. III. The experimental setup is described in Sec. IV. A novel multichannel imaging spectroscopic system is described in detail in Sec. V. Electron densities, flow velocities, and electron temperatures are measured simultaneously at multiple locations in the plasma jet using the spectroscopic system. The theory of Stark broadening is reviewed to explain the principle of the plasma density diagnostics in Sec. VI. A simple method for obtaining the plasma density from spectral line profiles is given. Experimental results are presented in Sec. VII and are compared to the predictions of the MHD pumping model in Sec. VIII. Section IX is a summary of the experimental results and analysis.

\section{MHD PUMPING AND COLLIMATION MECHANISM}

\section{A. Frozen-in flux: Magnetic Reynolds number}

The dynamics of magnetized plasmas is described by Maxwell's equations $\left(\nabla \times \boldsymbol{B}=\mu_{0} \boldsymbol{J} ; \nabla \cdot \boldsymbol{B}=0 ; \nabla \times \boldsymbol{E}=-\partial \boldsymbol{B} / \partial t\right)$ and Ohm's law $(\boldsymbol{J}=\sigma(\boldsymbol{E}+\boldsymbol{U} \times \boldsymbol{B}))$, where $\sigma$ is the electrical conductivity and $\boldsymbol{U}$ is the plasma flow velocity. The displacement current term is ignored in the $\nabla \times \boldsymbol{B}$ equation provided that the characteristic speed is much less than the speed of light. From these equations, the rate of growth of magnetic field strength can be derived:

$$
\begin{aligned}
\frac{\partial \boldsymbol{B}}{\partial t} & =-\nabla \times\left(\frac{\boldsymbol{J}}{\sigma}-\boldsymbol{U} \times \boldsymbol{B}\right) \\
& =\frac{-1}{\mu_{0} \sigma} \nabla \times \nabla \times \mathbf{B}+\nabla \times(\mathbf{U} \times \mathbf{B}) \\
& =\frac{1}{\mu_{0} \sigma} \nabla^{2} \mathbf{B}+\nabla \times(\mathbf{U} \times \mathbf{B}) .
\end{aligned}
$$

The first term (diffusion term) on the right-hand side represents the rate of diffusion of magnetic fields through plasma due to the finite electrical resistivity $\eta=1 / \sigma$. The second term (convection term) represents the rate of change of the magnetic field strength due to the plasma flow. In ideal MHD $(\boldsymbol{E}+\boldsymbol{U} \times \boldsymbol{B}=0)$, the diffusion term vanishes and the magnetic flux is frozen into the frame of the plasma flow. ${ }^{7}$

The magnitude of the convection term relative to the diffusion term determines whether the magnetic field at a point in the fluid may build up fast enough before it diffuses into the surroundings. The condition for magnetic field building up by fluid motion is that the frozen-in flux term is much greater than the diffusion term:

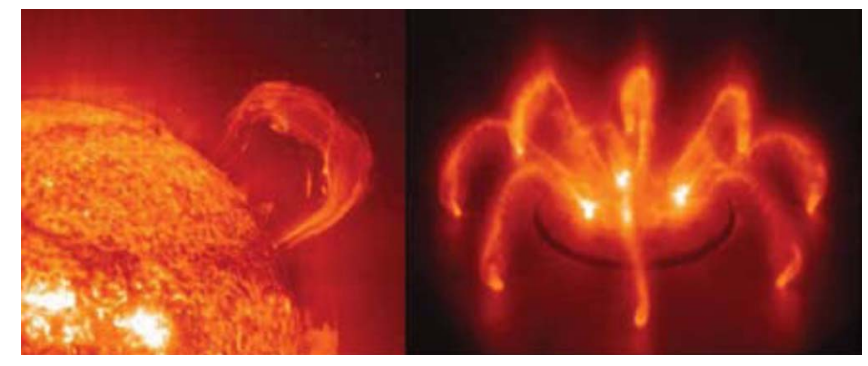

(a) Solar prominence

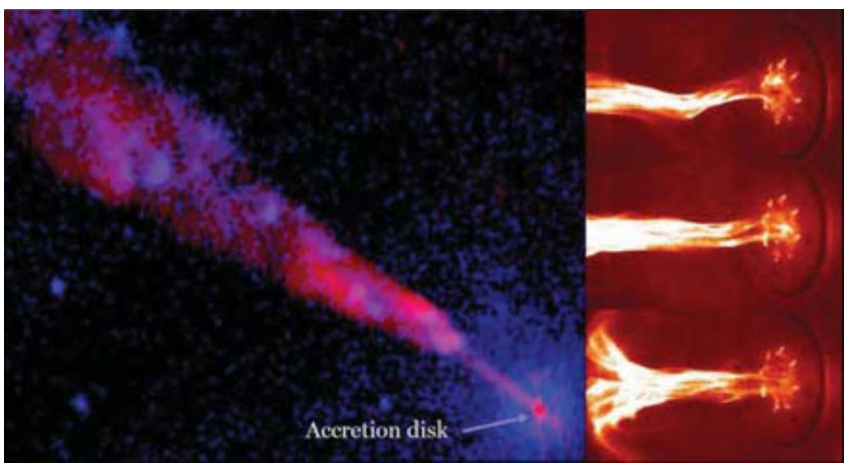

(b) Astrophysical jet

FIG. 1. (Color online) (a) Solar prominence (left; image from the Transition Region and Coronal Explorer) compared with the laboratory plasma arches (right). (b) Astrophysical jet (left; Centaurus A galaxy; Chandra x-ray/radio image) compared with the laboratory plasma jet (right).

$$
\begin{aligned}
\|\nabla \times(\boldsymbol{U} \times \boldsymbol{B})\| & \sim \frac{B}{L / U} \gg\left\|\frac{1}{\mu_{0} \sigma} \nabla^{2} \mathbf{B}\right\| \sim \frac{B}{\mu_{0} \sigma L^{2}} \\
& \Rightarrow \mu_{0} \sigma U L \gg 1,
\end{aligned}
$$

where $L$ is the characteristic length over which magnetic field varies.

Hence, the quantity $\mu_{0} \sigma U L$, called the magnetic Reynolds number $\left(R_{m}\right)$, is of fundamental importance in magnetized plasma. For plasmas with a small magnetic Reynolds number, there is no large gradient of magnetic fields in the plasma and the action of magnetic fields can be considered like a viscous drag on the fluid due to induced eddy currents. For plasmas with a large magnetic Reynolds number, the magnetic flux is carried by the fluid. The action of magnetic fields, if they deviate from their vacuum state, can now be thought as if a lateral pressure $B^{2} / 2 \mu_{0}$ is applied normal to the lines of force and a longitudinal tension $B^{2} / 2 \mu_{0}$ is applied along the lines of force. Since $R_{m} \gg 1$ for the plasmas described here, the magnetic flux is frozen into the moving plasma.

\section{B. MHD pumping and collimation model}

Consider a cylindrical magnetic flux tube as illustrated in Fig. 2. Initially, the flux tube is flared in the middle and has no current, and the plasma particles are concentrated near the foot points. An electric potential is applied along the field lines and subsequently an axial current starts to ramp up. The 


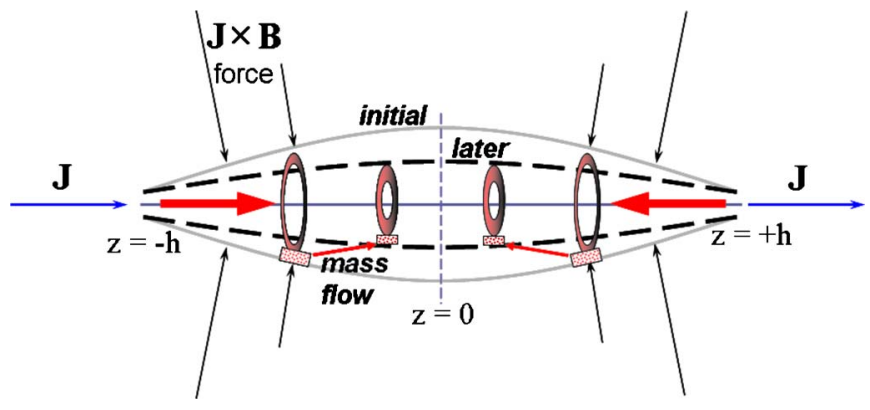

FIG. 2. (Color online) Collimation of initially flared flux tube (adapted from Ref. 5). Solid lines are initial magnetic flux surfaces and dashed lines are the same flux surfaces at a later time.

initial axial magnetic field $B_{z}$ is assumed to be strong enough to stabilize the flux tube against kink and sausage instabilities. The effect of $B_{z}$ on plasma instabilities and density scaling has been addressed elsewhere. ${ }^{6,8}$

The $\boldsymbol{J} \times \boldsymbol{B}$ force has a net axial component due to the flared geometry and creates axial flows:

$$
\begin{aligned}
\rho \frac{d U_{z}}{d t}= & (\boldsymbol{J} \times \boldsymbol{B})_{z}-\frac{\partial P}{\partial z}=J_{r} B_{\phi}-J_{\phi} B_{r}-\frac{\partial P}{\partial z} \\
= & -\frac{\partial}{\partial z}\left(\frac{B_{\phi}^{2}}{2 \mu_{0}}\right)-\frac{\partial P}{\partial z}-J_{\phi} B_{r}=-\frac{\partial}{\partial z}\left(\frac{B_{\phi}^{2}}{2 \mu_{0}}+P\right) \\
& -J_{\phi} B_{r} \\
\left(\because \mu_{0} J_{r}=\right. & \left.-\frac{\partial B_{\phi}}{\partial z} \text { due to the cylindrical symmetry }\right),
\end{aligned}
$$

where $U_{z}$ is the $z$-directed flow velocity, $\rho$ is the mass density, and $B, J$, and $P$ are the magnetic field strength, current density, and pressure, respectively.

The radial component of the $\boldsymbol{J} \times \boldsymbol{B}$ force describes the pinch force. The pinch force is greater near the foot points since both the current density and the magnetic field strength are greater there than in the middle. We assume that there is radial force balance so

$$
\frac{\partial P}{\partial r}=-J_{z} B_{\phi}
$$

We also assume that the current density $J_{z}$ is uniform inside a cross section of the flux tube, i.e., $J_{z}(z, r)=J_{z}(z)$ $=I_{0} / \pi a(z)^{2}$, where $I_{0}$ is the total current flowing in the flux tube and the local tube radius $a(z)$ describes the flaring of the flux tube. Then,

$$
\begin{aligned}
& B_{\phi}=\frac{\mu_{0}\left(J_{z} \pi r^{2}\right)}{2 \pi r}=\frac{\mu_{0} J_{z} r}{2}, \\
& \frac{\partial P}{\partial r}=-J_{z} B_{\phi}=-\frac{\mu_{0} J_{z}^{2} r}{2}, \\
& P=-\frac{\mu_{0} J_{z}^{2}}{4}\left(r^{2}-a^{2}\right)=\frac{B_{\phi}^{2}}{\mu_{0}}\left(\frac{a^{2}}{r^{2}}-1\right) .
\end{aligned}
$$

Thus, Eq. (3) becomes

$$
\begin{aligned}
\rho \frac{d U_{z}}{d t} & =-\frac{\partial}{\partial z}\left[\frac{B_{\phi}^{2}}{\mu_{0}}\left(\frac{a^{2}}{r^{2}}-\frac{1}{2}\right)\right]-J_{\phi} B_{r} \\
& =-\frac{\partial}{\partial z}\left[\frac{B_{\phi, a}^{2}}{\mu_{0}}\left(1-\frac{r^{2}}{2 a^{2}}\right)\right]-J_{\phi} B_{r},
\end{aligned}
$$

where $B_{\phi, a}=\mu_{0} I_{0} / 2 \pi a$ is the azimuthal magnetic field at the flux tube radius $a$. Near the axis, $J_{\phi} B_{r}$ and $r^{2} / a^{2}$ are very small, yielding an approximate expression

$$
\rho \frac{d U_{z}}{d t} \approx-\frac{\partial}{\partial z}\left[\frac{B_{\phi, a}^{2}}{\mu_{0}}\right] \text {. }
$$

Thus, the magnetic energy density term $B_{\phi, a}^{2} / \mu_{0}$ acts like an effective potential and so the plasma particles will move axially falling down this potential. Equation (9) can also be expressed in terms of the flux tube flaring $(\partial a / \partial z)$,

$$
\rho \frac{d U_{z}}{d t} \approx-\frac{\partial}{\partial z}\left[\frac{\mu_{0} I_{0}^{2}}{4 \pi^{2} a^{2}}\right]=\frac{\mu_{0} I^{2}}{2 \pi^{2} a^{3}} \frac{\partial a}{\partial z} .
$$

The potential $B_{\phi, a}^{2} / \mu_{0}$ has its minimum at the midaxial point of the flux tube due to the flared geometry of the flux tube. Therefore, counterstreaming axial flows will be generated from both foot points toward the middle, which has been verified experimentally by Stenson and Bellan. ${ }^{9}$ The counterstreaming flows collide with each other and so stagnate in the middle, resulting in accumulation of plasma particles there. By the frozen-in flux condition, the azimuthal magnetic flux carried by the flows also accumulates in the middle, which increases the azimuthal magnetic field strength $B_{\phi}$. As a result, the pinch force $J_{z} B_{\phi}$ increases and squeezes the magnetic flux tube in the middle. Thus, the magnetic flux radius becomes uniform axially, i.e., the magnetic flux tube becomes collimated. It should be noted that being collimated is the assumed initial state for kink and sausage instabilities and so consideration of collimation involves behavior prior to the occurrence of kink or sausage instabilities.

\section{CALTECH SPHEROMAK PLASMA GUN}

The Caltech spheromak plasma gun used in this work provides the physical boundary conditions for plasma-filled flux tubes, similar to the cases of astrophysical plasma jets and solar prominences. The adjective "spheromak" is used because the plasma gun had been used to study the spheromak formation mechanism in previous experiments ${ }^{4,6}$ and because the physics of plasma-filled flux tube collimation is related to the physics of spheromak formation. Spheromaks ${ }^{7}$ are a naturally occurring plasma confinement structure relevant to solar prominences. ${ }^{10}$ Spheromaks are one of the Taylor-relaxed states described by the force-free condition $\nabla \times \boldsymbol{B}=\lambda \boldsymbol{B}$, where the constant eigenvalue $\lambda$ is determined by the geometrical shape of the boundary confining the spheromak.

The most common and least complex method to generate spheromaks is to use a magnetized coaxial gun. ${ }^{11}$ The device is composed of a pair of concentric cylindrical electrodes (inner and outer) linked by a vacuum magnetic field produced by an external coil, as shown schematically in 


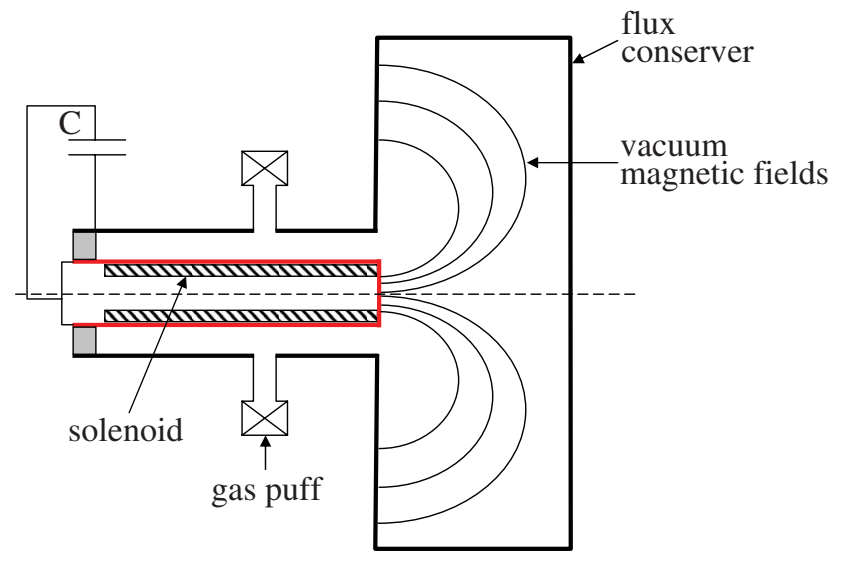

(a) Coaxial gun

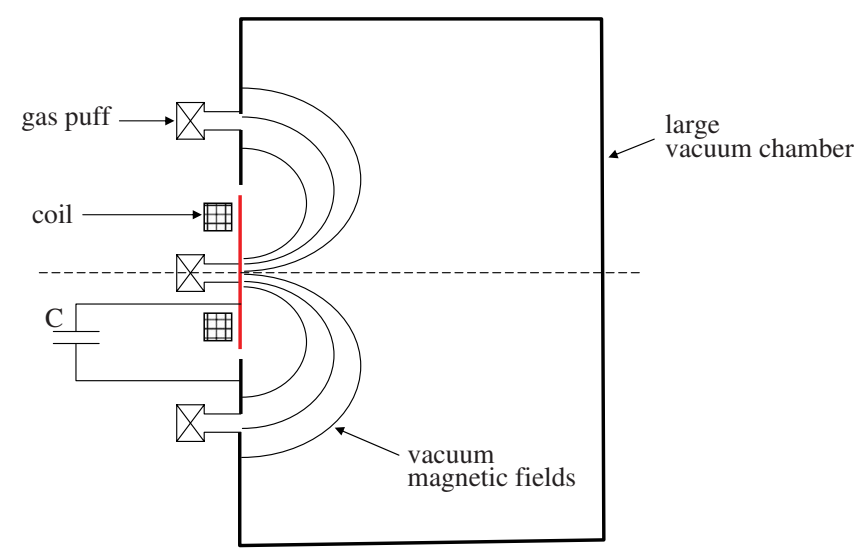

\section{(b) Planar coaxial gun}

FIG. 3. (Color online) Schematic drawings of (a) conventional cylindrical coaxial magnetized gun and (b) planar coaxial magnetized gun.

Fig. 3(a). The eigenvalue $\lambda$ of the spheromak produced by the coaxial gun is related to the total gun current $I_{\text {gun }}$ and the total magnetic flux $\psi_{\text {gun }}$ intercepting the inner electrode of surface area $S_{\text {gun }}$ as

$$
\lambda_{\text {plasma }}=\frac{\|\nabla \times \boldsymbol{B}\|}{B}=\frac{\mu_{0} J}{B}=\frac{\mu_{0} J S_{\text {gun }}}{B S_{\text {gun }}} \sim \frac{\mu_{0} I_{\text {gun }}}{\psi_{\text {gun }}} \equiv \lambda_{\text {gun }} .
$$

A modified coaxial gun design composed of planar electrodes instead of cylindrical electrodes was proposed by Bellan and constructed by Hsu and Bellan ${ }^{4,6}$ [see Fig. 3(b)]. The planar configuration has three main advantages: (1) The planar gun has larger area and thus requires smaller magnetic field to provide the same $\psi_{\text {gun. }}$. (2) The larger planar gun has a better matching between the source $\lambda_{\text {gun }}$ and the plasma $\lambda_{\text {plasma }}$, resulting in less plasma instability. ${ }^{7}$ (3) Absence of a flux conserver and the planar geometry of the electrodes provide better diagnostic access to the entire plasma evolution including the source gun region. In particular, direct imaging of the gun-produced plasmas became possible and has revealed the unique dynamics of the plasma structures. Hsu and Bellan ${ }^{6}$ showed that low $\lambda_{\text {gun }}$ values result in a straight

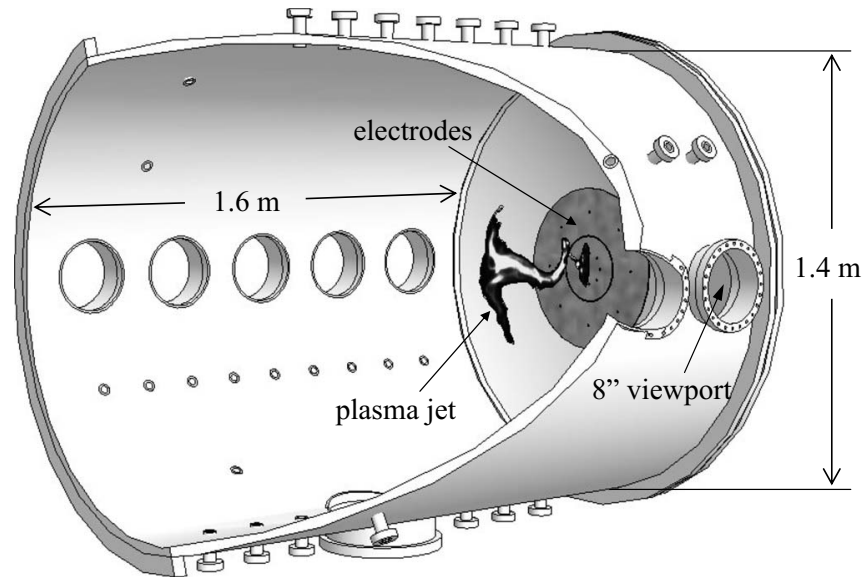

FIG. 4. Vacuum chamber. Total volume is $\sim 2.4 \mathrm{~m}^{3}$ and the base pressure is $1-2 \times 10^{-7}$ mbar. The chamber dimensions are much greater than the plasma. An image of a kinked plasma jet is inset for size comparison. The plasma expands freely without interaction with the chamber wall.

plasma jet, intermediate values lead to kinking of the jet axis (kink instability) in agreement with the Kruskal-Shafranov limit, and high values lead to a detachment of the plasma from the electrodes. Preliminary results suggested that breakoff of the jet from the electrodes due to a sausage instability occurring on top of the kink is a precursor to spheromak formation.

\section{EXPERIMENTAL SETUP}

The experimental setup comprises five major components: vacuum chamber, spheromak plasma gun, gas injection system, timing system, and diagnostics. These components are summarized here and a more detailed description can be found elsewhere. ${ }^{12}$

The vacuum chamber shown schematically in Fig. 4 provides a free space into which plasma can evolve. The plasma structures evolve without much interaction with the chamber wall because the plasma dimensions are much smaller than the chamber dimensions. This is in contrast to other spheromak experiments where the plasma geometry and the magnetic field configuration are essentially constrained by the chamber wall. The base pressure is $1-2 \times 10^{-7}$ mbar corresponding to a particle density of $\sim 5 \times 10^{15} \mathrm{~m}^{-3}$ at room temperature.

The spheromak plasma gun produces magnetized plasma structures. The plasma gun is mounted on the north end dome of the chamber, as shown in Fig. 4. The plasma gun comprises cathode and anode electrodes, gun capacitor bank, bias flux system, and gas feed lines. The cathode (inner electrode) is a disk copper plate and has eight gas orifices evenly spaced in a circle. The anode (outer electrode) is an annular copper plate and is coaxial and coplanar with the cathode. The anode also has eight orifices at locations corresponding to the cathode orifices. The small annular gap $(6 \mathrm{~mm})$ between the two electrodes is insulating at the low vacuum condition. High voltage $(3-8 \mathrm{kV})$ is applied across the electrodes using a $120 \mu \mathrm{F}$ capacitor bank switched by ignitrons. ${ }^{13}$ The gun bank supplies a current of the order of 60-150 kA flowing through the plasma. The bias coil 


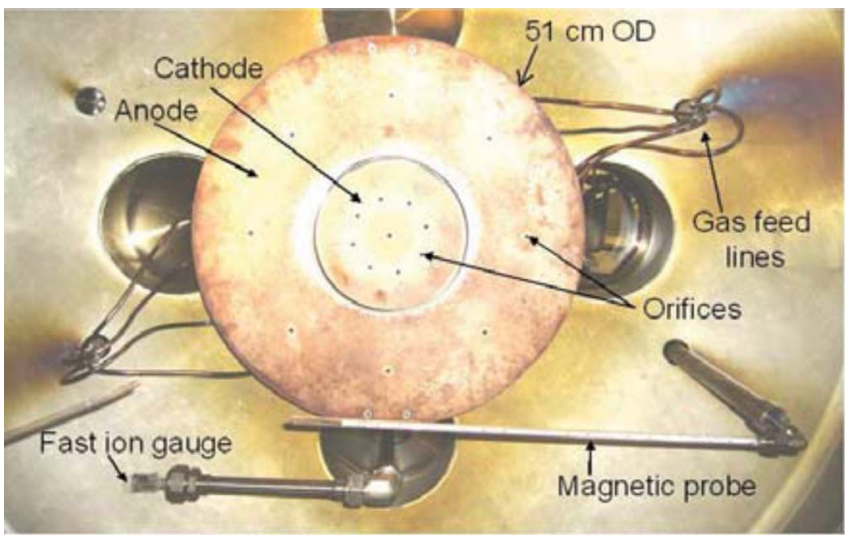

(a) front view

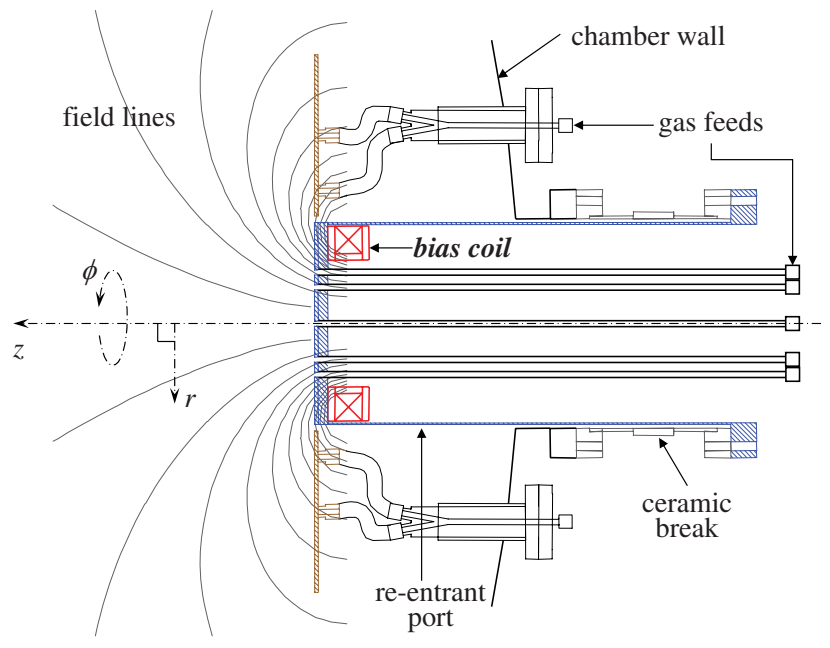

(b) side view (schematic)

FIG. 5. (Color online) Caltech spheromak plasma gun.

mounted on the rear side of the inner electrode produces an axisymmetric bias poloidal field, as illustrated in Fig. 5(b). The time scale of the coil current $(\sim 10 \mathrm{~ms})$ is much greater than the plasma lifetime $(\sim 20 \mu \mathrm{s})$ so that the bias field is constant over the duration of the plasma.

The gas injection system delivers high pressure gas into the vacuum on a millisecond time scale. The timing system consists of two independent subtiming modules. The main timing module triggers various power supplies for the plasma gun and the gas injection. The diagnostic timing module triggers diagnostic devices synchronized relative to plasma breakdown.

A variety of diagnostic devices are implemented in the experiment: a B-dot probe array for magnetic field measurements, Rogowski coils for gun current measurements, high speed cameras for imaging the evolving plasma structure, a $\mathrm{HeNe}$ laser heterodyne interferometer ${ }^{14}$ for line-averaged density, and a multichannel imaging spectroscopic system for density and flow velocity. The spectroscopic system is described in detail in Sec. V.

A distinctive plasma structure forms as the gas breaks

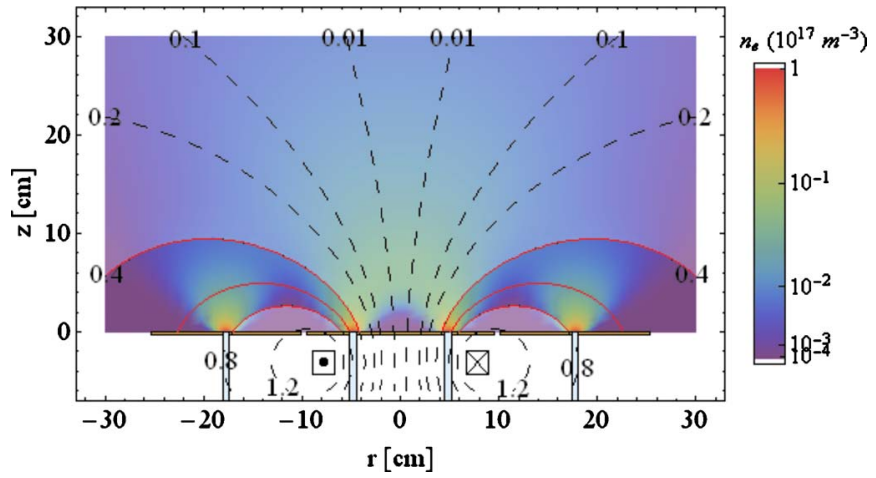

FIG. 6. (Color online) Neutral gas density profile and bias field lines (dashed lines) at the gas breakdown (adapted from Ref. 15). Each field line is labeled by the total poloidal flux (in milliweber) enclosed by the circle whose radius is from the axis of symmetry to the field line. Colors represent $n_{e}$ in a logarithmic scale as shown in the scale bar. Solid lines define the flux tubes where inside the gas will break down. The density profile in the flux tubes is highlighted.

down upon triggering the bias flux system, the gas injection system, and the gun bank in sequence. Figure 6 illustrates a typical profile of gas cloud and bias field at the instant of the breakdown. ${ }^{15}$ The initial plasma structure is defined by eight flux tubes indicated by red solid field lines in the figure. Each flux tube spans a gas orifice on the cathode and the corresponding orifice on the anode, indicating that the Paschen breakdown condition is optimal along the flux tube path. The ionized gas particles are pumped into the flux tubes from the source gas orifices by MHD force, ${ }^{15}$ forming a distinctive structure reminiscent of spider legs, as shown in Fig. 7. Subsequent evolution of the spider legs is characterized by formation of a collimating plasma jet.

\section{MULTICHANNEL IMAGING SPECTROSCOPY SYSTEM}

Shifts, broadenings, and relative intensities of atomic spectral lines emitted by plasma reflect the physical condition of the plasma such as flow velocities $(U)$, electron densities $\left(n_{e}\right)$, and electron, ion temperatures $\left(T_{e}, T_{i}\right)$. Spectral emissions from moving particles are shifted and broadened by the Doppler effect. The average Doppler shift is proportional to the flow velocity $U$. Strong line broadening $(>1 \mathrm{~nm})$ can occur for hydrogenic spectral lines via collective Stark effects of electrons and ions. Stark-broadened hydrogen lines are routinely used for $n_{e}$ diagnostics. ${ }^{16}$ Stark broadening is relatively weak for nonhydrogenic lines but can become significant $(>0.1 \mathrm{~nm})$ and stronger than Doppler broadening for $n_{e} \gg 10^{20} \mathrm{~m}^{-3}$ and $T_{e}<10 \mathrm{eV}$. A vast amount of literature on the theory of the Stark broadening ${ }^{17-19}$ is available but may not be comprehensible to nonexperts due to the mathematical complexity. We provide an abbreviated explanation of the Stark broadening from first principles in Sec. VI for the sake of completeness. In the case of optically thin plasma in local thermodynamic equilibrium (LTE), i.e., collisional depopulation rate $\gg$ radiative decay rate, the relative line intensities are described by the Saha-Boltzmann relation, which is a strong 


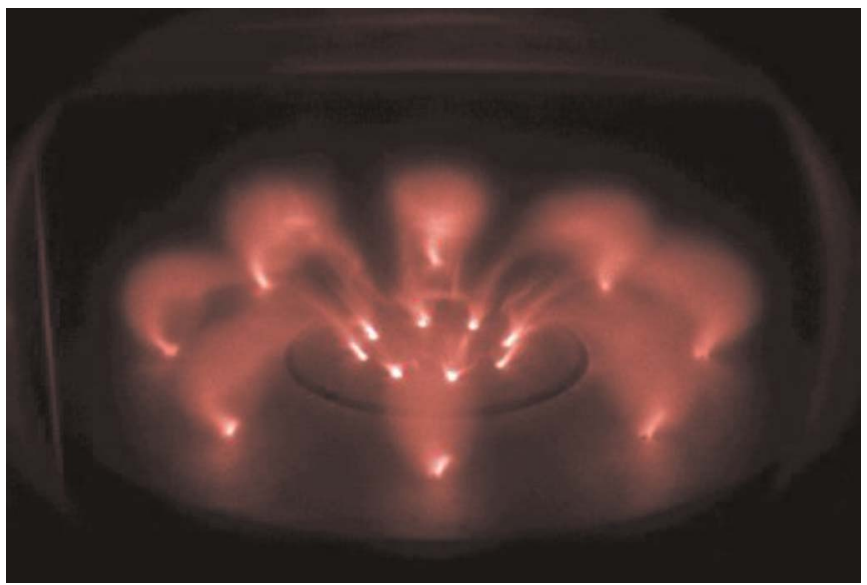

FIG. 7. (Color online) Eight plasma-filled current-carrying flux tubes (shot 6529: $\mathrm{N}_{2}$ inner+Ne outer). This distinctive plasma structure reminiscent of spider legs is formed by breaking down the gas cloud shown in Fig. 6.

function of $T_{e}$. The validity criteria ${ }^{20}$ of the partial LTE for the principal quantum number $p$ are numerically given by

$$
p \gtrsim 700\left(\frac{T_{e}}{n_{e}^{2}}\right)^{1 / 17} Z^{14 / 17} \text { and } p \geq 2\left(\frac{Z^{2}}{T_{e}}\right)^{1 / 2},
$$

where $n_{e}$ is in $\mathrm{m}^{-3}, T_{e}$ is in $\mathrm{eV}$, and $\mathrm{Z}$ is the effective nuclear charge experienced by the valence electron. Many spectral lines with $p \geq 2$ emitted by our plasma $\left(n_{e} \geq 10^{22} \mathrm{~m}^{-3}\right.$ and $\left.T_{e}<10 \mathrm{eV}\right)$ satisfy the partial LTE conditions.

Despite ample diagnostic information contained in spectral emissions, interpretation of spectral line profiles is often obscured by various effects such as instrumental broadenings, plasma inhomogeneity, and uncertainty in lines of sight for the case of highly dynamic plasma structures. Furthermore, irreproducibility of detailed plasma structure in our experiments can invalidate the comparison of spectral profiles from multiple locations if measurements are made on separate discharges.

A multichannel high-resolution imaging spectroscopic system has been constructed in order to overcome these issues. The spectroscopic system can measure spectral emissions at multiple locations of the plasma at the same time with definite lines of sight. The spectroscopic system, illustrated in Fig. 8, consists of a camera lens, a 12-channel fiber array, entrance optics, a Czerny-Turner-type spectrometer, and a gated intensified charge-coupled device (ICCD). The camera lens forms an image of plasma on the plane of the fiber array input. The plasma emission is intercepted by the fiber array at 12 different locations and fed into the spectrometer. The emission spectra analyzed by the spectrometer are then recorded by the ICCD detector. The ICCD detector is triggered and gated by the diagnostic timing system which is synchronized with plasma breakdown by optically coupling the intense radiation at the time of breakdown. This enables the system to take spectral measurements with submicrosecond time resolution.

The specifications of the spectroscopic system are summarized here and further details can be found elsewhere. ${ }^{12}$ (1) The wavelength range is $200-500 \mathrm{~nm}$. (2) The spectral resolution, bandpass (=pixel resolution $\times$ number of pixels),

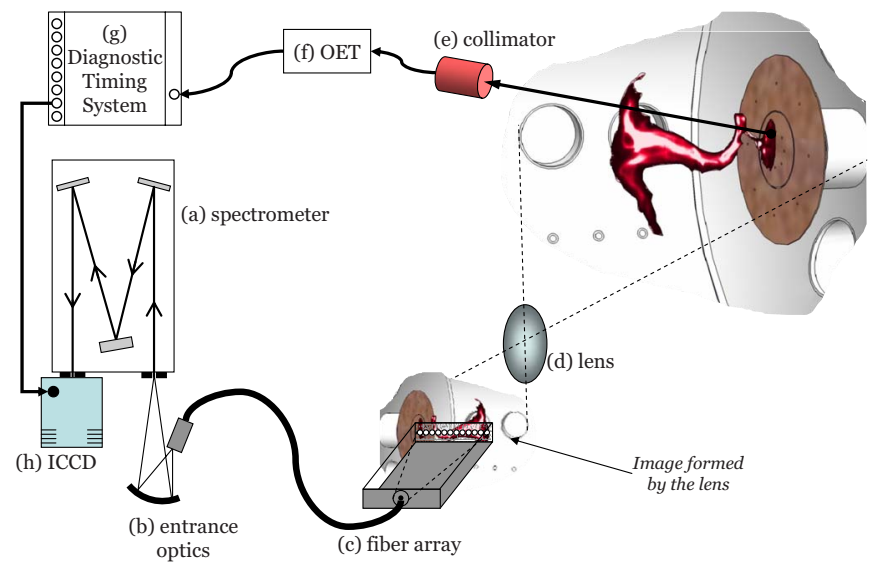

FIG. 8. (Color online) Schematic drawing of the spectroscopic system. The system consists of (a) Czerny-Turner spectrometer (JY Horiba 1000M: $1 \mathrm{~m}$ focal length, f/8 aperture, 3600 grooves/mm grating), (b) F/\# matching entrance optics, (c) 12-channel linear fiber assembly [UV/visible grade, 0.22 NA (NA denotes numerical aperture), $100 \mu \mathrm{m}$ core size, $10 \mathrm{~m}$ long], (d) camera lens, (e) collimator (Fiberguide MACRO collimator; $22.2 \mathrm{~mm}$ aperture, $0.22 \mathrm{NA}$ ), (f) optoelectric transducer, (g) diagnostic timing system, and (h) ICCD detector (Andor DH520-25F-03; 16 bpp dynamic range, $800 \times 256$ active pixels, $26 \mu \mathrm{m}$ square pixel).

and the Doppler shift corresponding to one pixel shift are $\sim 6 \mathrm{pm} / \mathrm{pixel}, \sim 5 \mathrm{~nm}$, and $\sim 5 \mathrm{~km} / \mathrm{s}$, respectively. These are determined by ICCD pixel size and spectrometer parameters. ${ }^{12}$ (3) The temporal resolution is about $0.3 \mu \mathrm{s}$. The best temporal resolution is achieved by matching f-numbers between optical components and thereby increasing signal-to-noise ratio at the ICCD detector. (4) The spatial resolution is $\sim 3-5 \mathrm{~mm}$, which is determined by the diameter of the line-of-sight volume of each fiber. The space between adjacent lines of sight is $\sim 30-50 \mathrm{~mm}$. Lines of sight are illustrated in Fig. 9.

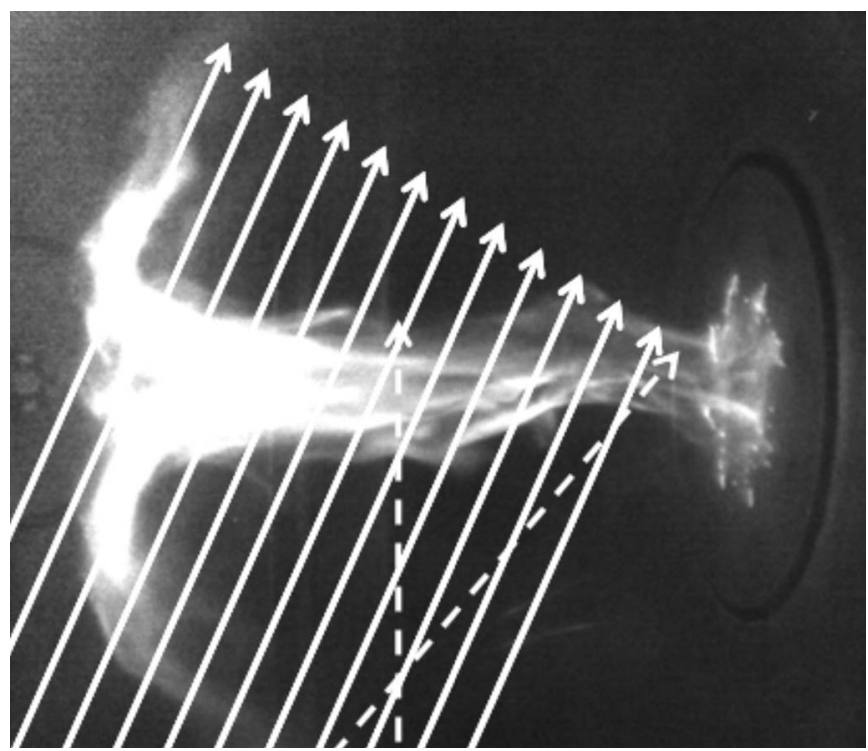

FIG. 9. (Solid lines) Lines of sight of the fiber array intercept the plasma jet at approximately equally spaced locations. (Dashed lines) In some experiments, the fiber array is replaced by a bundle of multiple fibers to obtain multiple line-of-sight angles. 


\section{DENSITY DIAGNOSIS USING STARK BROADENING}

The Stark effect is the splitting and shifting of a spectral line in the presence of an electric field. The Stark effect differs significantly among spectral lines and can be divided into two categories according to its dependence on the applied electric field, namely, the quadratic Stark effect and the linear Stark effect.

The quadratic Stark effect results from the perturbing electric field on nondegenerate energy levels and can be understood using perturbation theory. For an atom having a single electron or a single valence electron in its outermost shell subject to a uniform electric field $F$ in the positive $z$-direction, the change in the energy level of the triple quantum number $(n, l, m)$ state is given by (up to the second order in $F$ )

$$
\begin{aligned}
\Delta E_{n l m} \simeq & e F\langle n, l, m|Z| n, l, m\rangle+e^{2} F^{2} \\
& \times \sum_{n^{\prime}} \sum_{l^{\prime}=l \pm 1} \frac{\left|\left\langle n, l, m|Z| n^{\prime}, l^{\prime}, m\right\rangle\right|^{2}}{E_{n l m}-E_{n^{\prime} l^{\prime} m}} \\
\equiv & -\frac{1}{2} \alpha_{n l m} F^{2},
\end{aligned}
$$

where the linear term in $F$ vanishes by the selection rules ${ }^{12}$ for $l \neq 0$ and the spherical symmetry for $l=0$, and $\alpha_{n l m}$ is the polarizability of the eigenstate $|n l m\rangle$. For example, the polarizability of the ground state $(n=1)$ of the hydrogen atom (this is the only nondegenerate hydrogen state) is $\alpha_{100}=18 \pi \epsilon_{0} a_{0}^{3}$, where $a_{0}$ is the Bohr radius.

For an $N_{n}$-fold degenerate energy level $E_{n}$, each degenerate state can be represented as a combination of eigenstates of the perturbation term $e F Z$, which can make the linear term finite, i.e.,

$$
\Delta E_{n g} \approx e \lambda_{n g} F,
$$

where $g$ represents one of the $N_{n}$ degenerate eigenstates and $\lambda_{n g}$ is the eigenvalue of the operator $Z$. As an example, the $n=2$ hydrogen state (fourfold degenerate) will split into three levels: $\Delta E_{2}=0, \pm 3 a_{0} e F$. The linear Stark effect is usually much stronger than the quadratic Stark effect. The Stark effect is linear for hydrogenic lines and quadratic for nonhydrogenic lines in general.

In a plasma environment, a radiating atom or ion (emitter) is under the influence of the local electric fields produced by the surrounding ions and electrons within the Debye sphere of the emitter. The collective Stark effect due to these local electric fields results in broadening of spectral lines, i.e., Stark broadening. The emitter experiences two very different electric fields, the quasistatic electric field due to slow moving ions and the transient electric field due to fast moving electrons. The quasistatic electric field splits a degenerate atomic level of the emitter into distinct individual components called Stark components. The Stark components are then broadened by the transient electric field due to electron impacts. If the atomic level is not degenerate, the level undergoes only the electron impact broadening. The resulting line profile will be the sum of all possible transitions from the Stark components of an upper level to the Stark components of a lower level. In a real plasma, the line profile is

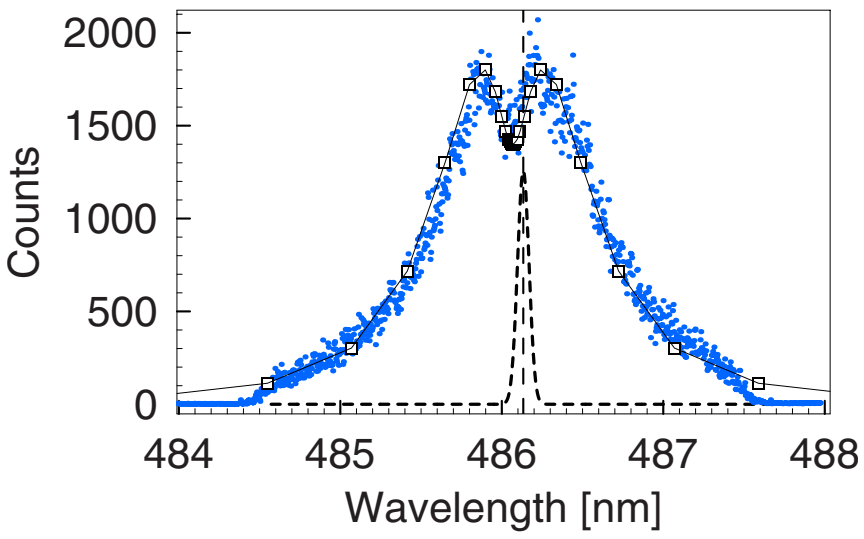

FIG. 10. (Color online) $\mathrm{H}_{\beta}$ profile showing the characteristic central dip (shot oma11.28; spectrometer ICCD exposure $=1 \mu \mathrm{s}$ ). The square points are the theoretical Stark profile for $n_{e}=1.3 \times 10^{22} \mathrm{~m}^{-3}$ and $T=2 \mathrm{eV}$ according to Stehlé (Ref. 24). A Doppler profile (dashed line) for $T_{i}=5 \mathrm{eV}$ at the $\mathrm{H}_{\beta}$ rest-frame wavelength $(486.133 \mathrm{~nm})$ is also shown for comparison with the much broader Stark profile. The estimated Stark FWHM according to Eq. (17) is $\sim 1.1 \mathrm{~nm}$, corresponding to $n_{e} \sim 1.2 \times 10^{22} \mathrm{~m}^{-3}$. Note that the data points near the profile tail ends are flat since they are outside the bandpass of the spectroscopic system.

further modified by secondary effects such as Debye screening ${ }^{21}$ and ion motion, ${ }^{22-24}$ which are complicated functions of plasma density and temperature. However, for density diagnosis, the detail of the Stark broadened profile is not necessarily required. Instead, the full width at half maximum (FWHM) of the profile is used because the electron density is a simple function of the Stark FWHM for many spectral lines.

For hydrogen lines, the profile is dominantly broadened by the splitting of degenerate energy levels by the ion quasistatic electric field via the strong linear Stark effect. The profile width scales with the electron density as ${ }^{16}$

$$
w_{S}=2.5 \times 10^{-14} \alpha_{1 / 2} n_{e}^{2 / 3},
$$

where $w_{S}$ is the Stark FWHM in nanometers, $n_{e}$ is the electron density in $\mathrm{m}^{-3}$, and the parameter $\alpha_{1 / 2}$ is the so called reduced half-width. The half-width $\alpha_{1 / 2}$ has been tabulated for many hydrogen lines based on both theoretical calculations and experimental data ${ }^{21,22,24}$ for the temperature range of $0.5-4 \mathrm{eV}$ and density range of $10^{20}-10^{24} \mathrm{~m}^{-3}$. In particular, the $\mathrm{H}_{\beta}$ line has been the most popular choice for density diagnosis because its Stark width is nearly independent of plasma temperature. Figure 10 is an example of the $\mathrm{H}_{\beta}$ line observed in our experiments. The characteristic central dip indicates the energy level splitting by the quasistatic electric field.

For lines of nonhydrogenic atoms, the electron impact broadening via the quadratic Stark effect is the dominant broadening mechanism. ${ }^{16}$ To a first approximation, the line profile is Lorentzian and linearly proportional to the electron density,

$$
w_{S} \approx w_{m} n_{e}
$$

where $w_{m}$ is the proportionality parameter. For an extensive collection of $\mathrm{N}_{\text {II }}$ lines, this property has been substantiated by the experimental work of Mar et $a .^{25}$ Measurements of 


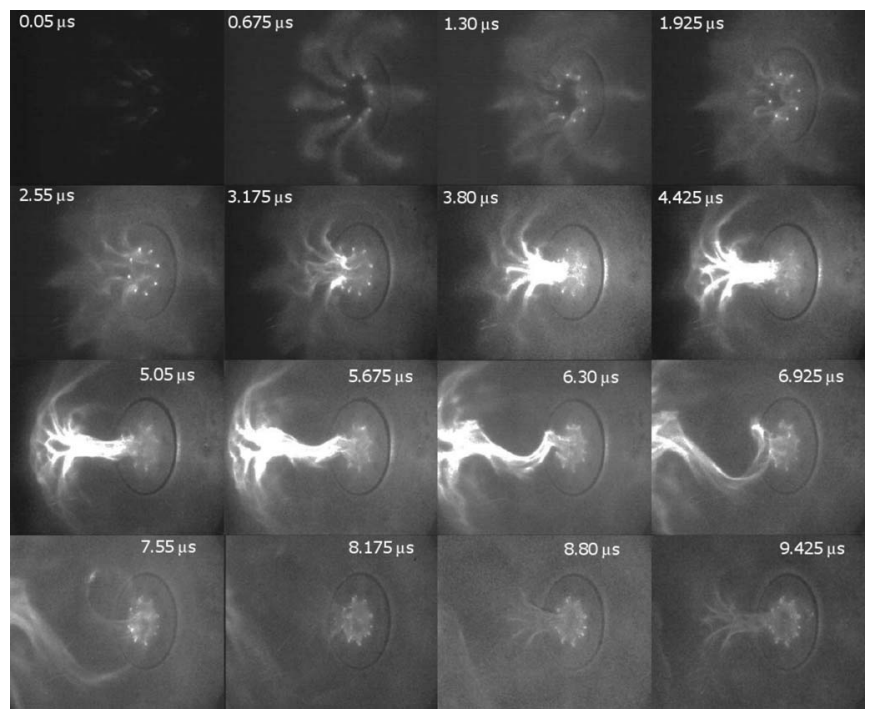

FIG. 11. Evolving plasma structure (shot 7217, hydrogen plasma). The frame sequence is from left to right and top to bottom. The delay of the first frame is $0.05 \mu$ s with respect to breakdown and the interframe delay is $0.625 \mu \mathrm{s}$. In the last few frames, notice the presence of ghosting from previous frames.

Stark broadening of nonhydrogenic spectral lines have been tabulated for well-defined and independently measured plasma conditions. ${ }^{26}$

A simple and computationally inexpensive procedure is used in our work to extract the pure Stark width from a line profile and then estimate the electron density using Eqs. (15) and (16). The total FWHM $\left(w_{T}\right)$ of a spectral line profile is measured by fitting the data to a Lorentzian profile. The Stark FWHM $\left(w_{S}\right)$ is then obtained by subtracting the Doppler effect $\left(w_{D}\right)$ and the instrumental function $\left(w_{I}\right)$ from the total width $w_{T}$ according to the conventional method ${ }^{27}$

$$
w_{S}=\sqrt{w_{T}^{2}-w_{D}^{2}-w_{I}^{2}} \quad \text { if } w_{T} / \sqrt{w_{D}^{2}+w_{I}^{2}}>1.04 .
$$

This formula assumes that the instrumental function is Gaussian, the Stark broadening is Lorentzian, and so the total broadening is a Voigt profile, ${ }^{28}$ i.e., the convolution of a Gaussian profile and a Lorentzian profile. However, the validity of this simple procedure is in question in case of hydrogen lines emitted from high density plasma $\left(n_{e} \gtrsim 10^{22} \mathrm{~m}^{-3}\right)$ because the line profiles can be significantly different from the Lorentzian function. The reliability of the

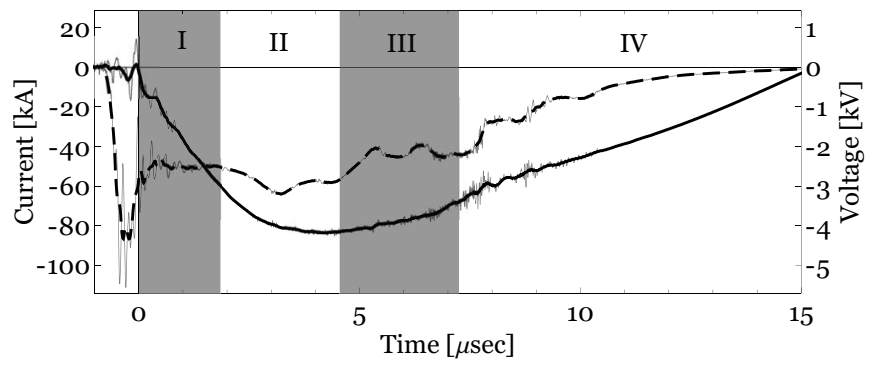

FIG. 12. Gun current trace (solid curve) and voltage trace (dashed curve). Data correspond to the same plasma discharge (shot 7217) as in Fig. 11. Times are with respect to breakdown. Roman numerals indicate the evolution stages.

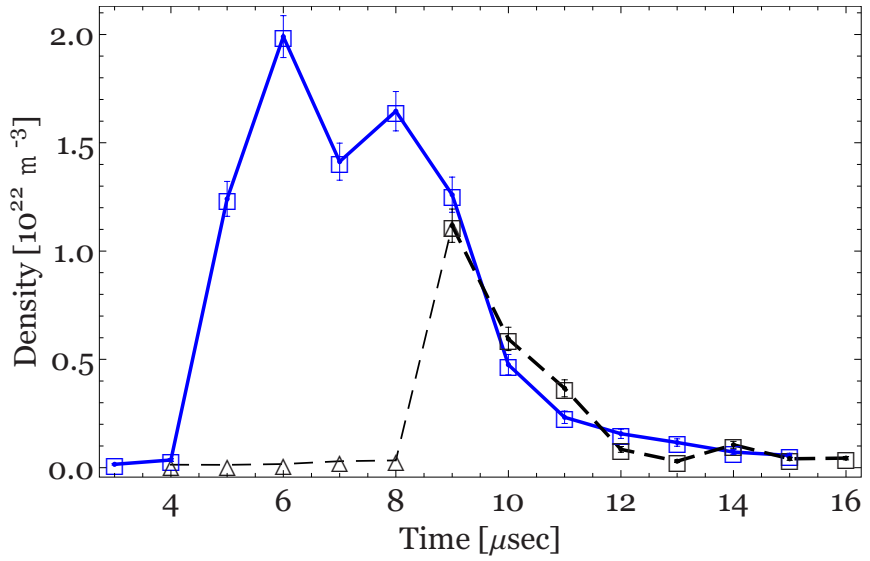

FIG. 13. (Color online) Electron density of a hydrogen plasma jet vs time (shot range: oma11.16-69). Density is estimated from the $\mathrm{H}_{\beta}$ line profiles. Squares correspond to the solid (oblique) line of sight and triangles to the dashed (perpendicular) line of sight in Fig. 9. For the perpendicular line of sight, the density is zero initially and appears suddenly at $t=9 \mu$ s as expected because the jet takes time to reach the line-of-sight volume. This is consistent with the measured jet velocity $(\sim 30 \mathrm{~km} / \mathrm{s})$.

simple method is substantiated by comparing the density obtained by the simple method with the density obtained by the theoretical fitting according to Stehlé ${ }^{24}$ (see Fig. 10). The uncertainty in the density estimation using $\mathrm{H}_{\beta}$ profiles is less than $15 \%$ for $n_{e} \geq 10^{22} \mathrm{~m}^{-3}$, taking into account various issues ${ }^{12}$ such as plasma inhomogeneity, Zeeman effect, internal relative flow effect, and uncertainties in $w_{D}$ and Stark parameters $\left(\alpha_{1 / 2}\right.$ or $\left.w_{m}\right)$. The relative uncertainty gets larger for lower density: $\sim 25 \%$ for $n_{e} \sim 10^{21} \mathrm{~m}^{-3}$ and $\sim 50 \%$ for $n_{e} \sim 10^{20} \mathrm{~m}^{-3}$.

\section{EXPERIMENTAL RESULTS}

Following gas breakdown, the plasma expands into vacuum undergoing several structural changes, as shown in the fast camera images (Fig. 11). The evolution of the plasma has four distinctive stages: (i) formation and collimation of spider legs (frames 1 and 2), (ii) coalescing of spider legs

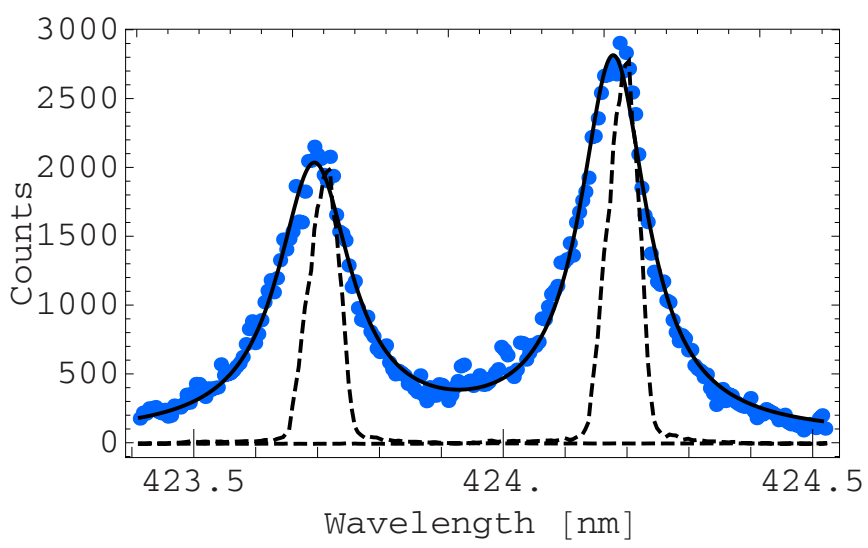

FIG. 14. (Color online) Stark broadened $\mathrm{N}_{\mathrm{II}}$ spectral lines from a nitrogen plasma jet (shot 7245; spectrometer ICCD exposure $=0.1 \mu \mathrm{s}$ ). The restframe wavelengths of the two spectral lines are 423.705 and $424.178 \mathrm{~nm}$, respectively. Instrumental function (dashed line) is shown at the two restframe wavelengths for width comparison. Solid line is a Lorentzian curve fit to the spectrum. Estimated density is $\sim 8 \times 10^{22} \mathrm{~m}^{-3}$. 


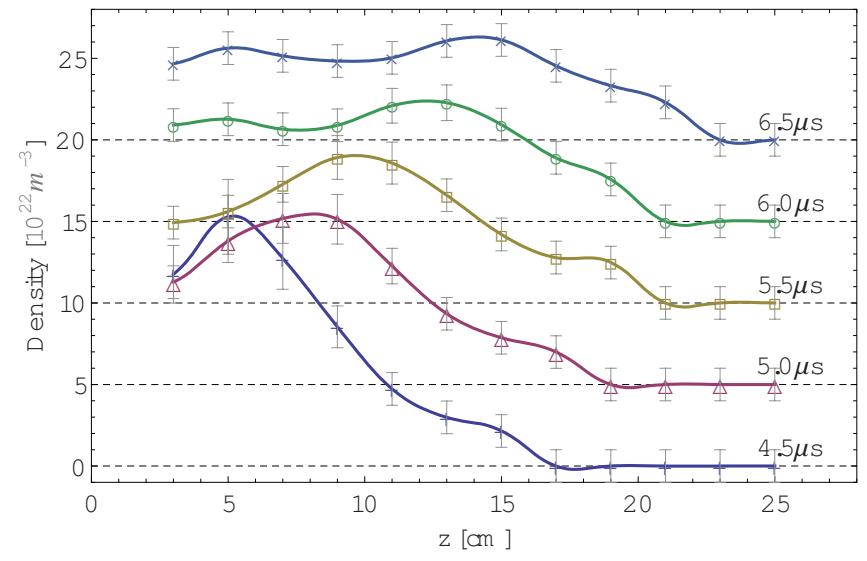

(a) Density profile

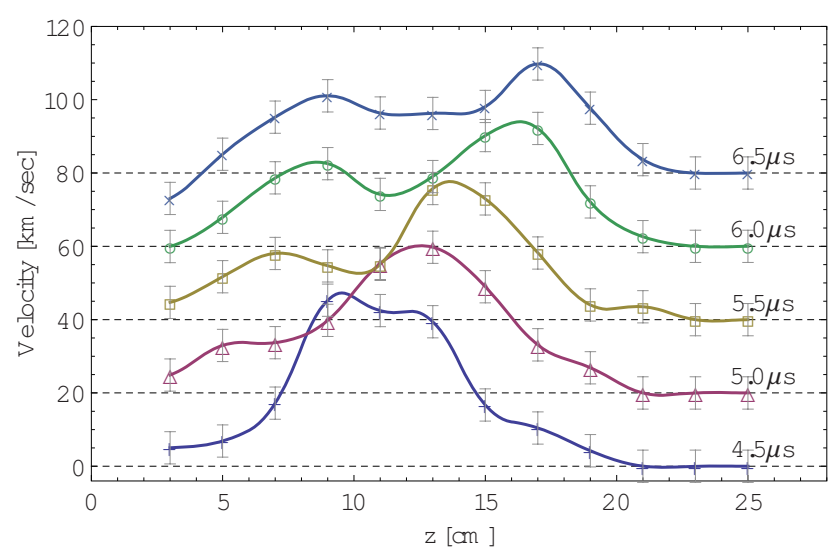

(b) Velocity profile

FIG. 15. (Color online) (a) Density and (b) velocity along the axis of a straight column nitrogen plasma jet (shot range: 8209-8227). Densities were estimated from Stark broadened $\mathrm{N}_{\text {II }} 404.131 \mathrm{~nm}$ lines. Velocities were estimated from the Doppler shifts of the same $\mathrm{N}_{\text {II }}$ lines. The line-of-sight angle $\left(\sim 60^{\circ}\right)$ and the "blue" Stark shifts (Ref. 26) $\left(\sim 0.8\right.$ pm per $\left.10^{22} \mathrm{~m}^{-3}\right)$ are taken into account in the velocity measurement. Solid curves are polynomial fits to the data and dashed horizontal lines indicate zero lines. Each data point is an average of three measurements. Measurement errors are the larger of $\pm 1 \times 10^{22} \mathrm{~m}^{-3}$ or $\pm 15 \%$ for the density and $\pm 5 \mathrm{~km} / \mathrm{s}$ for the velocity.

into a central plasma jet (frames 3-6), (iii) expansion and collimation of plasma jet (frames 7-10), and (iv) instability of plasma jet and spheromak formation (frames 11-16). It is interesting to note that, in contrast with the substantial structural changes of the plasma, the current and voltage traces are almost featureless, as shown in Fig. 12. The first and the last stages were studied in detail in previous work, ${ }^{6,15}$ and the present work focuses on the third stage.

\section{A. Observation of large density amplification}

Electron densities were measured from the Stark broadened spectral lines by the method described in Sec. VI. The time dependence of the electron density of a hydrogen plasma jet is shown in Fig. 13. Peak density of the order of $10^{22} \mathrm{~m}^{-3}$ is observed when the spider legs merge com-
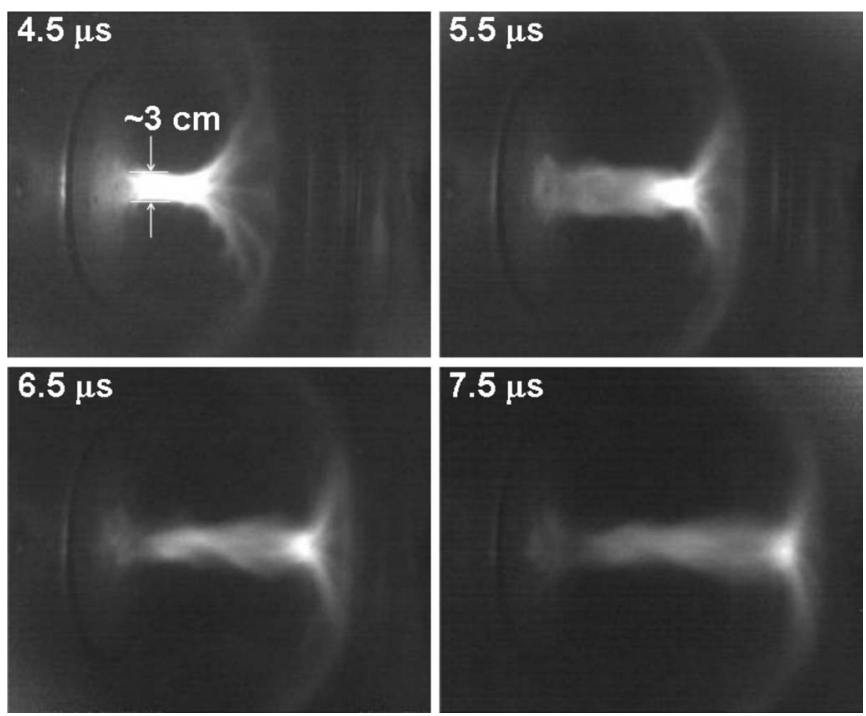

FIG. 16. Nitrogen plasma jet (shot 8209). The bright blob propagates with an Alfvénic velocity of $\sim 40 \mathrm{~km} / \mathrm{s}$ catching up the expanding outskirts of the jet.

pletely. The density is only $\sim 10^{20} \mathrm{~m}^{-3}$ when the plasma starts to form the central jet but then increases by a factor of 100 when the jet fully forms. Merging of the spider legs does not decrease the total volume of the plasma and thus cannot account for this large density amplification. On the other hand, the observed density amplification is consistent with the particle ingestion and flux tube collimation process described in the MHD pumping model.

Similar density amplification is observed in nonhydrogen plasmas. For nitrogen plasma jets, Stark broadened $\mathrm{N}_{\mathrm{II}}$ spectral lines are used for density estimation and a typical emission spectrum is shown in Fig. 14. Nitrogen plasma jets show even greater peak density of $\sim 1 \times 10^{23} \mathrm{~m}^{-3}$.

\section{B. Axial profiles of density and velocity}

Densities along the axis of a straight nitrogen plasma jet were measured at several different timings. The axial density profile has a sharp peak initially but becomes uniform as the jet expands axially, as can be seen in Fig. 15(a). Velocity profiles were measured from the Doppler shifts of the same spectra used in the density measurement and are shown in Fig. 15(b). The velocity peak is always ahead of the density peak by $3-5 \mathrm{~cm}$ in space and by $\sim 1 \mu \mathrm{s}$ in time. Negative velocity gradient (i.e., slowing down of the flow) is clearly seen near the jet front.

A detailed comparison with the camera images (Fig. 16) shows that the bright blob in the jet coincides with the region between the density peak and the velocity peak. The blob propagates along the jet axis with an Alfvénic velocity of $\sim 40 \mathrm{~km} / \mathrm{s}$. The blob is faster than the expanding outskirts of the jet and catches up with it later, as seen in the last frame of Fig. 16. This observation is consistent with the negative velocity gradient near the jet front.

For the detailed analysis in Sec. VIII, three density pro- 

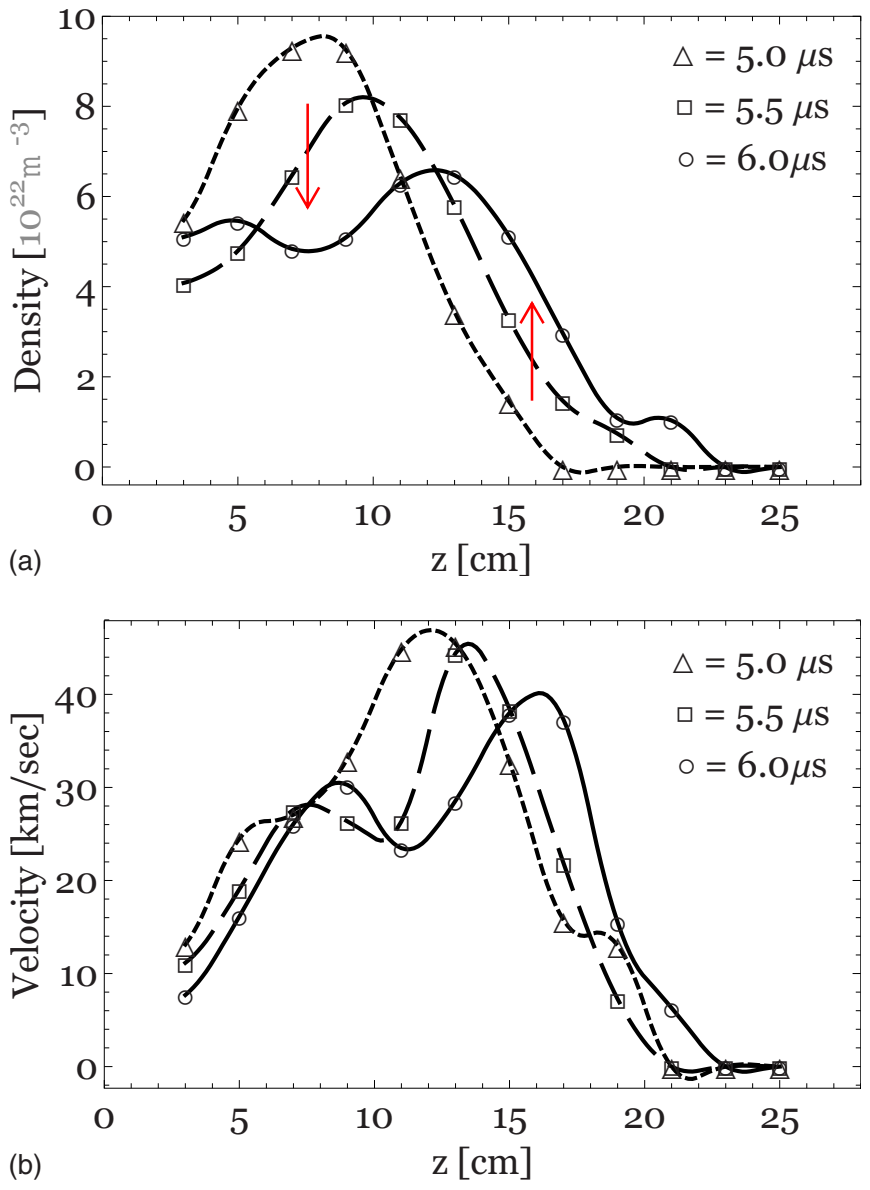

FIG. 17. (Color online) Detailed view of density (top) and velocity (bottom) profiles. The density increases near the jet front and becomes uniform in the main body of the jet as indicated by arrows.

files are selected from Fig. 15(a) and shown overlaid in Fig. 17(a). The corresponding velocity profiles are shown overlaid in Fig. 17(b).

\section{Axially uniform $T_{e}$}

As discussed in Sec. V, our plasmas satisfy the partial LTE conditions. ${ }^{20}$ Thus, electron temperatures $\left(T_{e}\right)$ can be estimated by comparing the measured spectrum containing several atomic lines of the same species with the calculated spectrum using the Saha-Boltzmann relation and the transition probabilities of the atomic levels. The relative line ratios were obtained $^{29}$ for a range of $T_{e}$ values $(0.5-10 \mathrm{eV})$ with the $n_{e}$ taken from the Stark broadening measurement. The instrumental function was convoluted with the line ratios to obtain more realistic spectra. $T_{e}$ was then determined by finding the best fit to the measurement. An example is shown in Fig. 18 for a nitrogen spectrum. In this example, the estimated $T_{e}$ is 2-4 eV, where the uncertainty can be improved by applying the same process to multiple spectral bands.

Uniform $T_{e} \sim 3 \mathrm{eV}$ along the jet axis was observed except near the electrode $z<5 \mathrm{~cm}$, where the LTE temperature is ill defined. The plasma jet is expected to be axially isothermal since thermal electrons can move quickly along the magnetic field line.

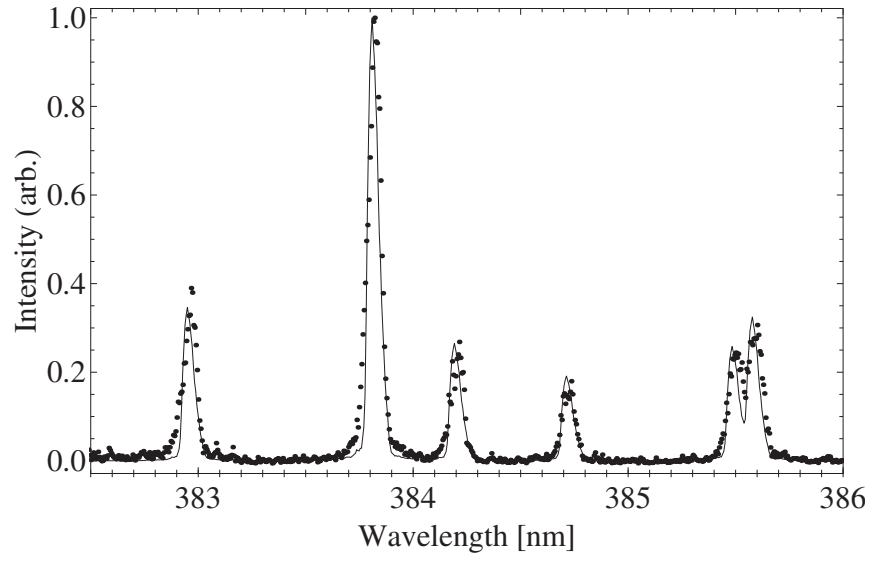

FIG. 18. Relative spectral line intensities from a nitrogen plasma jet (shot 7265). Dots are the measurement and solid line is the calculated Saha-LTE spectrum for $T_{e}=3 \mathrm{eV}$ and $n_{e} \sim 6 \times 10^{22} \mathrm{~m}^{-3}$.

\section{DISCUSSION}

\section{A. MHD pumping}

The Stark broadening density measurements show that the plasma jets become very dense on a microsecond time scale. This result is supported by an independent density measurement using a heterodyne HeNe interferometer. ${ }^{8}$ The density of the pre-breakdown neutral gas, as measured by a fast ion gauge, ${ }^{15}$ is only $10^{17} \mathrm{~m}^{-3}$. The measured peak density of the nitrogen plasma jet is of the order of $10^{23} \mathrm{~m}^{-3}$ at the same location $6 \mu$ s later, so the particle density has increased by a factor of $10^{6}$. This steep density rise cannot be explained by a pinch effect associated with the gun current flowing in the jet flux tube. The merging of the spider legs cannot account for the peak density of the jet, either, since the spider leg density $\left(\sim 10^{21} \mathrm{~m}^{-3}\right)$ is 100 times less than the jet density and the total volume of the spider legs is smaller than the volume of the central jet. Plasma particles must be pumped into the flux tubes from the source gas orifices.

According to the MHD pumping model, the driving force of the plasma jet dynamics is the axial gradient of the azimuthal magnetic energy associated with the axial current [see Eq. (9)],

$$
\rho \frac{d U_{z}}{d t} \approx-\frac{\partial}{\partial z}\left[\frac{B_{\phi, a}^{2}}{\mu_{0}}\right],
$$

where $B_{\phi, a}=\mu_{0} I_{0} / 2 \pi a$ is the azimuthal magnetic field at the flux tube radius $a$. The effective potential $B_{\phi, a}^{2} / \mu_{0}$ has negative slope everywhere because $B_{\phi, a}$ decreases along the axis due to the flared geometry of the jet flux tube. Thus, the plasma will fall down the slope and gain kinetic energy as it moves. The order of magnitude of the kinetic energy gain will be

$$
\rho U_{z}^{2} \sim-\Delta\left(\frac{B_{\phi, a}^{2}}{\mu_{0}}\right) \approx\left(\frac{B_{\phi, a}^{2}}{\mu_{0}}\right)_{z=0} .
$$

Using $\rho=m_{i} n_{e}$ yields $n_{e} \sim\left(B_{\phi, a}^{2} / \mu_{0}\right)_{z=0} / m_{i} U_{z}^{2}$, where $m_{i}$ is the ion mass. For nitrogen plasma jets, the observed $U_{z} \sim 40 \mathrm{~km} / \mathrm{s}$ and $B_{\phi, a} \sim 1.0 \mathrm{~T}$ (using $I_{0} \approx 100 \mathrm{kA}$ and $a(z=0) \approx 2 \mathrm{~cm})$ give an electron density $n_{e}$ of the order of 
$10^{22} \mathrm{~m}^{-3}$, which is consistent with the Stark broadening density measurement. Equation (19) suggests that $U_{z} \propto B_{\phi, a} \propto I_{0}$ if the density $\rho$ is independent of the total current $I_{0}$. Kumar and Bellan ${ }^{8}$ have verified this scaling relation by measuring $U_{z}$ and $n_{e}$ using the heterodyne interferometer.

\section{B. MHD collimation}

However, the collimation of the axially expanding jet cannot be explained by this consistency check alone. Since the collimation is associated with slowing down of flow and accumulation of particles according to the MHD pumping model, the observed features of the density and velocity profiles in Fig. 17 are now examined using the continuity equation

$$
\frac{\partial \rho}{\partial t}=-\nabla \cdot(\rho \boldsymbol{U})=-U_{z} \frac{\partial \rho}{\partial z}-\rho \frac{\partial U_{z}}{\partial z}-\nabla_{\perp} \cdot\left(\rho \boldsymbol{U}_{\perp}\right),
$$

where $U_{z} \partial \rho / \partial z$ represents the density change due to the axial convection, negative $\rho \partial U_{z} / \partial z$ corresponds to an axial compression, and negative $\nabla_{\perp} \cdot\left(\rho \boldsymbol{U}_{\perp}\right)$ corresponds to a radial pinching. Since the diameter of the jet remains approximately constant during the axial expansion, the radial pinching effect can be ignored for the moment, yielding

$$
\frac{\partial \rho}{\partial t} \approx-U_{z} \frac{\partial \rho}{\partial z}-\rho \frac{\partial U_{z}}{\partial z} .
$$

Suppose there is no flow gradient, i.e., $\partial U_{z} / \partial z=0$. Then, $\partial \rho / \partial t \approx-U_{z} \partial \rho / \partial z$, which can be integrated to give $\rho(z, t)=\rho\left(z-U_{z} t\right)$. Thus, the axial density profile would just translate without any deformation if there is no flow gradient. Our plasma jets show a large negative flow gradient $\left(\partial U_{z} / \partial z \ll 0\right)$ at the jet front and so the density increases in that region because

$$
\left.\frac{\partial \rho}{\partial t} \approx-\rho \frac{\partial U_{z}}{\partial z} \gg 0 \quad \text { (at the jet front }\right) .
$$

The observed density increase at the jet front is indicated by an arrow in Fig. 17(a). The observation that the density peak always appears at $\sim 1 \mu$ s after the velocity peak also conforms with the continuity equation because (1) at the location of the velocity peak, $\partial U_{z} / \partial z=0$ and $\partial \rho / \partial z<0$ (see Fig. 17), giving

$$
\frac{\partial \rho}{\partial t} \approx-U_{z} \frac{\partial \rho}{\partial z}>0 \quad \text { (near the velocity peak), }
$$

and (2) on the other hand, at the density peak, $\partial \rho / \partial z=0$ and $\partial U_{z} / \partial z>0$, giving

$$
\frac{\partial \rho}{\partial t} \approx-\rho \frac{\partial U_{z}}{\partial z}<0 \quad \text { (near the density peak) } .
$$

The collimation of the axially expanding jet can be summarized as follows according to the MHD pumping model. The axial component of the $\boldsymbol{J} \times \boldsymbol{B}$ force accelerates the jet into the flared magnetic flux tube produced by the bias coil. The flow velocity slows down near the jet front where the flux tube radius is large. The slowing down of the flow results in accumulation of mass $(\partial \rho / \partial t>0)$ as well as the azi- muthal magnetic flux carried by the mass flow. As the magnetic flux accumulates, the magnetic field intensity increases and so does the pinch force. The amplified pinch force subsequently squeezes the flared jet front $\left(\nabla_{\perp} \cdot \boldsymbol{U}_{\perp}<0\right)$. This squeezing action at the jet front continues while the jet expands axially into the flared flux tube, resulting in an elongated collimated jet.

The density becomes axially uniform in the main body of the jet as it becomes collimated. The cause of the flow deceleration has not yet been investigated but could be the tension of the curved bias magnetic field lines or buildup of neutral particles swept by the jet at the flow front.

\section{Total particle flux by the MHD pumping}

The total particle flux ingested into the jet is approximately

$$
\left(\frac{d N_{\mathrm{T}}}{d t}\right)_{\mathrm{MHD}}=\pi a^{2} n_{e} U_{z} \sim 10^{18} \text { particles } / \mu \mathrm{s},
$$

using $a \sim 3 \mathrm{~cm}, n_{e} \sim 10^{22} \mathrm{~m}^{-3}$, and $U_{z} \sim 30 \mathrm{~km} / \mathrm{s}$. This MHD pumping speed is much faster than the gas puffing, ${ }^{15}$ $d N_{\mathrm{T}} / d t \approx 10^{16} \mu \mathrm{s}^{-1}$. Integrating over the plasma duration of $\sim 10 \mu$ s (which is limited by the capacitance of the main gun bank), the total number of particles ingested by the MHD pumping is $N_{\mathrm{T}, \mathrm{MHD}} \sim 10^{19}$. This amounts to about $20 \%$ of the total number of puffed particles as estimated by the fast ion gauge measurement. ${ }^{15}$

\section{SUMMARY}

We have demonstrated that the MHD pumping and collimation model provides a consistent explanation for the observed collimation and density amplification of the gun-produced plasmas. The measured jet velocities $(\sim 40 \mathrm{~km} / \mathrm{s})$ are the Alfvénic velocity corresponding to the measured density $\left(\sim 10^{22} \mathrm{~m}^{-3}\right)$ and azimuthal magnetic field strength $(\sim 1 \mathrm{~T}$ near the gun electrode) as predicted by the MHD pumping model. It is demonstrated that the flow slowing down observed at the jet forefront leads to the observed mass accumulation and subsequent jet collimation according to the MHD pumping model. In addition, the measured density amplification (from $\sim 10^{17}$ up to $\sim 10^{22} \mathrm{~m}^{-3}$ ) is entirely due to the MHD pumping and cannot be accounted by the gas puffing.

\section{ACKNOWLEDGMENTS}

This work was supported by U.S. DOE (Grant No. DEF602-04ER54755).

${ }^{1}$ W. H. T. Vlemmings, P. J. Diamond, and H. Imai, Nature (London) 440, 58 (2006).

${ }^{2}$ Y. Kato, Astrophys. Space Sci. 307, 11 (2007).

${ }^{3}$ E. R. Priest, Dynamics and Structure of Quiescent Solar Prominences, 2nd ed. (Kluwer, Dordrecht, 1989).

${ }^{4}$ S. C. Hsu and P. M. Bellan, Mon. Not. R. Astron. Soc. 334, 257 (2002).

${ }^{5}$ P. M. Bellan, Phys. Plasmas 10, 1999 (2003).

${ }^{6}$ S. C. Hsu and P. M. Bellan, Phys. Rev. Lett. 90, 215002 (2003).

${ }^{7}$ P. M. Bellan, Spheromaks (Imperial College Press, London, 2000).

${ }^{8}$ D. Kumar and P. M. Bellan, Phys. Rev. Lett. 103, 105003 (2009).

${ }^{9}$ E. V. Stenson and P. M. Bellan, IEEE Trans. Plasma Sci. 36, 1206 (2008).

${ }^{10}$ P. M. Bellan and J. F. Hansen, Phys. Plasmas 5, 1991 (1998). 
${ }^{11}$ H. Alfvén, L. Lindberg, and P. Mitlid, J. Nucl. Energy, Part C 1, 116 (1960).

${ }^{12}$ G. S. Yun, "Dynamics of plasma structures interacting with external and self-generated magnetic fields," Ph.D. thesis, California Institute of Technology, 2008.

${ }^{13}$ D. Kumar, A. L. Moser, and P. M. Bellan, IEEE Trans. Plasma Sci. 38, 47 (2010).

${ }^{14}$ D. Kumar and P. M. Bellan, Rev. Sci. Instrum. 77, 083503 (2006).

${ }^{15}$ S. You, G. S. Yun, and P. M. Bellan, Phys. Rev. Lett. 95, 045002 (2005).

${ }^{16}$ W. L. Wiese, Plasma Diagnostic Techniques (Academic, New York, 1965).

${ }^{17}$ H. Margenau and M. Lewis, Rev. Mod. Phys. 31, 569 (1959).

${ }^{18}$ V. S. Lisitsa, Sov. Phys. Usp. 20, 603 (1977).

${ }^{19}$ J. M. Luque, M. D. Calzada, and M. Sáez, J. Phys. B 36, 1573 (2003).
${ }^{20}$ T. Fujimoto and R. W. P. McWhirter, Phys. Rev. A 42, 6588 (1990).

${ }^{21}$ P. Kepple and H. R. Griem, Phys. Rev. 173, 317 (1968).

${ }^{22}$ M. A. Gigosos and V. Cardeñoso, J. Phys. B 29, 4795 (1996).

${ }^{23}$ C. Stehlé, Astron. Astrophys. Suppl. Ser. 104, 509 (1994).

${ }^{24}$ C. Stehlé and R. Hutcheon, Astron. Astrophys. Suppl. Ser. 140, 93 (1999).

${ }^{25}$ S. Mar, J. A. Aparicio, M. I. de la Rosa, J. A. del Val, M. A. Gigosos, V. R. González, and C. Pérez, J. Phys. B 33, 1169 (2000).

${ }^{26}$ N. Konjević, A. Lesage, J. R. Fuhr, and W. L. Wiese, J. Phys. Chem. Ref. Data 31, 819 (2002).

${ }^{27}$ M. Mimura and K. Sato, Jpn. J. Appl. Phys., Part 1 34, 1668 (1995).

${ }^{28}$ Y. Liu, J. Lin, G. Huang, Y. Guo, and C. Duan, J. Opt. Soc. Am. B 18, 666 (2001).

${ }^{29}$ Yu. Ralchenko, A. E. Kramida, J. Reader, and NIST ASD Team, NIST Atomic Spectra Database version 3.1.5 (2008). 